\section{CUTANEOUS IMMUNE-RELATED ADVERSE EVENTS ARE PROTECTIVE OF MORTALITY IN PATIENTS TREATED WITH ANTI-PD1 AND ANTI-PDL1 THERAPY IN A MULTI- INSTITUTIONAL COHORT STUDY}

${ }^{1}$ Yevgeniy Semenov*, ${ }^{2}$ Kimberly Tang, ${ }^{2}$ Jayhyun Seo, ${ }^{2}$ Kerry Reynolds, ${ }^{3}$ Bruce Tiu, ${ }^{4}$ Thomas Le, ${ }^{3}$ Vartan Pahalyants, ${ }^{2}$ Neel Raval, ${ }^{2}$ Pearl Ugwu-Dike, ${ }^{2}$ Leyre Zubiri, ${ }^{2}$ Vivek Naranbhai, ${ }^{5}$ Alexander Gusev, ${ }^{6}$ Nicole LeBoeuf, ${ }^{3}$ Maryam Asgari, ${ }^{4}$ Shawn Kwatra. 'Massachusetts General Hospital/Harvard Medical School, Boston, MA, MA, United States, ${ }^{2}$ Massachusetts General Hospital, Boston, MA, United States; ${ }^{3}$ Harvard Medical School, Boston, MA, United States: ${ }^{4}$ Johns Hopkins University, Baltimore, MD, United States; ${ }^{5}$ Dana Farber Cancer Institute, Boston, MA, United States; ${ }^{6}$ Dana Faber Cancer Institute, Boston, MA, United States

Background Immune checkpoint inhibitors (ICIs) have revolutionized cancer therapy over the last decade. Despite the efficacy of ICIs, immune-related adverse events (irAEs) occur in over a third of treated patients and can cause lasting morbidity and mortality. ${ }^{1-3}$ Cutaneous irAEs (cirAEs) are the most frequently reported toxicities, occurring in $20-40 \%$ of treated patients. Though recent reports have investigated the prognostic significance of irAEs on cancer outcomes, little is known about the specific impact of cirAEs and their subtypes on cancer survival. ${ }^{4}$ In this landmark analysis, we present the first population-level study examining the influence of cirAE development following ICI therapy on the mortality of cancer patients.

Methods 7,008 patients who developed cirAEs after treatment with anti-programmed cell death receptor/ligand 1 (PD-1/PDL1) therapy for malignant neoplasms of digestive organs, bronchus or lung, melanoma of skin, and urinary tract were identified through the TriNetX Diamond network along with 7,008 matched controls (table 1). The malignant neoplasms and cutaneous diagnoses for this study were identified from published literature and expert opinion. ${ }^{5}{ }^{6}$ Looking at cutaneous eruptions within 6 months of the first instance of ICI administration, a 6-month landmark analysis using a Cox proportional hazards model was performed to determine the impact of cirAE on overall survival.

Results Presence of any cirAE $(\mathrm{HR}=0.695, \mathrm{p}<0.0001)$, nonspecific rashes $(\mathrm{HR}=0.704, \mathrm{p}<0.0001)$, pruritus $(\mathrm{HR}=0.695$, $\mathrm{p}<0.0001)$, drug eruption $(\mathrm{HR}=0.755, \mathrm{p}=0.0013)$, and xerosis $(\mathrm{HR}=0.626, \mathrm{p}=0.0013)$ were significantly protective of mortality using a Benjamini-Hochberg $(\mathrm{BH})$ correction for multiple comparisons (table 2). Psoriasis $(\mathrm{HR}=0.703$, $\mathrm{p}=0.0451)$ and lichen planus/lichenoid dermatitis $(\mathrm{HR}=0.511$, $\mathrm{p}=0.0274$ ) were nominally significant. Notably, though not reaching statistical significance, eczematous dermatitis $(\mathrm{HR}=0.0 .612), \quad$ vitiligo $\quad(\mathrm{HR}=0.534), \quad$ bullous pemphigoid $(\mathrm{HR}=0.524)$, and Grover's disease $(\mathrm{HR}=0.468)$ were associated with strong protective clinical effects. To explore the impact of landmark time on mortality, a sensitivity analysis was performed for cirAE onset within 3 months $(\mathrm{HR}=0.759$, $\mathrm{p}<0.0001)$ and 9 months $(\mathrm{HR}=0.84, \mathrm{p}<0.0001)$ of ICI initiation. A separate sensitivity analysis expanded to include all cancer types treated with ICI yielded similar results, with additional statistical significance reached for ICI-induced psoriasis (table 3).
Conclusions This is the first population-level study and largest analysis to date of the impact of cirAEs on mortality among patients with advanced cancer. With the exception of mucositis and hyperhidrosis, there was a strong clinically protective effect of cirAEs across all individual morphologies investigated. Our results demonstrate that cirAE development after ICI initiation is an important positive prognostic indicator of response to ICI therapy and patient survival.

Abstract 814 Table 1 Propensity score-matched baseline characteristics for patients treated with PD-1 or PL-L1 therapy

\begin{tabular}{|c|c|c|c|c|c|}
\hline \multirow{3}{*}{$\begin{array}{l}\mathrm{N} \\
\text { Age (years) }\end{array}$} & \multicolumn{2}{|c|}{ ICI with cirAE } & \multicolumn{2}{|c|}{ ICI without cirAE } & \multirow[t]{2}{*}{ P-value } \\
\hline & & & & & \\
\hline & Mean & SD & Mean & SD & \\
\hline Age at Index & 68.2 & 11.2 & 68.3 & 11.1 & 0.7912 \\
\hline Gender & $\mathrm{N}$ & $\%$ & $\mathrm{~N}$ & $\%$ & \\
\hline Male & 3972 & 56.7 & 3961 & 56.5 & 0.8513 \\
\hline Female & 3036 & 43.3 & 3044 & 43.4 & 0.8915 \\
\hline Unknown & 0 & 0 & 10 & 0.14 & 0.0016 \\
\hline Race and Ethnicity & Mean & $\%$ & Mean & $\%$ & \\
\hline White Non-Hispanic & 1549 & 22.10 & 1571 & 22.40 & 0.6551 \\
\hline Black Non-Hispanic & 129 & 1.84 & 116 & 1.66 & 0.4021 \\
\hline Asian Non-Hispanic & 17 & 0.24 & 10 & 0.14 & 0.1775 \\
\hline Hispanic or Latino & 111 & 1.58 & 77 & 1.10 & 0.0125 \\
\hline Cancer Type & Mean & $\%$ & Mean & $\%$ & \\
\hline Digestive organs & 956 & 13.64 & 937 & 13.37 & 0.6387 \\
\hline Bronchus and lung & 3993 & 56.9 & 3996 & 57.02 & 0.9592 \\
\hline Melanoma of skin & 1300 & 18.55 & 1288 & 18.38 & 0.7939 \\
\hline Urinary tract & 1306 & 18.64 & 1244 & 17.75 & 0.1746 \\
\hline $\begin{array}{l}\text { Ill-defined, other } \\
\text { secondary and } \\
\text { unspecified sites }\end{array}$ & 5399 & 77.04 & 5396 & 76.99 & 0.952 \\
\hline
\end{tabular}

Abstract 814 Table 2 Association between cutaneous eruptions and surviv

\begin{tabular}{|c|c|c|c|}
\hline Cutaneous Diagnosis ${ }^{\ddagger}$ & $\mathbf{N}$ & Hazard Ratio & P-value \\
\hline Hyperhidrosis & 281 & 1.381 & 0.0797 \\
\hline Mucositis & 563 & 1.161 & 0.2068 \\
\hline Dermatomyositis & 105 & 0.93 & 0.7894 \\
\hline Maculopapular eruption & 230 & 0.845 & 0.3625 \\
\hline Erythroderma & 247 & 0.769 & 0.1697 \\
\hline $\begin{array}{l}\text { Drug eruption and non-specific drug } \\
\text { reaction }\end{array}$ & 1075 & 0.755 & 0.0013 \\
\hline Hyperkeratosis & 39 & 0.707 & 0.4867 \\
\hline Rash and other non-specific eruption & 3163 & 0.704 & $<0.0001$ \\
\hline Psoriasis & 299 & 0.703 & 0.0451 \\
\hline Pruritus & 1694 & 0.695 & $<0.0001$ \\
\hline Xerostomia & 163 & 0.671 & 0.1301 \\
\hline Xerosis & 441 & 0.626 & 0.0013 \\
\hline Eczema and Atopic Dermatitis & 72 & 0.612 & 0.1467 \\
\hline Vitiligo & 100 & 0.534 & 0.0929 \\
\hline Pemphigoid & 32 & 0.524 & 0.3281 \\
\hline Lichen Planus & 97 & 0.511 & 0.0274 \\
\hline Grover's disease & 18 & 0.468 & 0.2768 \\
\hline Any cutaneous diagnosis & 7008 & 0.778 & $<0.0001$ \\
\hline \multicolumn{4}{|c|}{$\begin{array}{l}{ }^{*} \text { Cutaneous diagnoses were identified based on published literature and expert } \\
\text { opinion. } \\
\text { "Benjamini-Hochberg p-value of significance }=0.0013 \text {. }\end{array}$} \\
\hline
\end{tabular}


Abstract 814 Table 3 Association between cutaneous eruptions and surviva

\begin{tabular}{lccc}
\hline Cutaneous Diagnosis & N & Hazard Ratio & P-value* \\
\hline Dermatomyositis & 128 & 1.405 & 0.1917 \\
\hline Mucositis & 694 & 1.112 & 0.3398 \\
\hline Hyperhidrosis & 322 & 1.063 & 0.697 \\
\hline Maculopapular eruption & 264 & 0.951 & 0.7777 \\
\hline Lichen Planus & 113 & 0.849 & 0.5988 \\
\hline $\begin{array}{l}\text { Drug eruption and non-specific drug } \\
\text { reaction }\end{array}$ & 1194 & 0.83 & 0.028 \\
\hline Erythroderma & 295 & 0.79 & 0.1833 \\
\hline Rash and other non-specific eruption & $\mathbf{3 5 5 7}$ & $\mathbf{0 . 7 6 9}$ & $<\mathbf{0 . 0 0 0 1}$ \\
\hline Hyperkeratosis & 48 & 0.741 & 0.5026 \\
\hline Pruritus & $\mathbf{1 9 0 1}$ & $\mathbf{0 . 7 3 7}$ & $<\mathbf{0 . 0 0 0 1}$ \\
\hline Xerosis & $\mathbf{4 9 9}$ & $\mathbf{0 . 6 5 8}$ & $\mathbf{0 . 0 0 2 5}$ \\
\hline Xerostomia & 228 & 0.63 & 0.0328 \\
\hline Eczema and Atopic Dermatitis & 83 & 0.628 & 0.1633 \\
\hline Psoriasis & $\mathbf{3 3 6}$ & $\mathbf{0 . 5 8 1}$ & $\mathbf{0 . 0 0 0 5}$ \\
\hline Grover's Disease & 21 & 0.481 & 0.2993 \\
\hline Vitiligo & 103 & 0.458 & 0.0285 \\
\hline Pemphigoid & 35 & 0.264 & 0.0207 \\
\hline Any cutaneous diagnosis & $\mathbf{7 9 7 5}$ & $\mathbf{0 . 8 0 5}$ & $<\mathbf{0 . 0 0 0 1}$ \\
\hline *Cutaneous diagnoses were identified based on published literature and expert \\
opinion.
\end{tabular}

"Benjamini-Hochberg p-value of significance $=0.0025$.

Cutaneous diagnoses were identified based on published literature and expert opinion. *Benjamini-Hochberg p-value of significance $=0.0025$.

\section{REFERENCES}

1. Puzanov I, Diab A, Abdallah K, et al. Managing toxicities associated with immune checkpoint inhibitors: consensus recommendations from the Society for Immunotherapy of Cancer (SITC) Toxicity Management Working Group. I Immunother Cancer 2017;5(1):1-28. doi:10.1186/s40425-017-0300-z.

2. Brahmer JR, Lacchetti C, Schneider BJ, et al. Management of immune-related adverse events in patients treated with immune checkpoint inhibitor therapy: American society of clinical oncology clinical practice guideline. J Clin Oncol 2018:36(17):1714-1768. doi:10.1200/JC0.2017.77.6385.

3. Phillips GS, Wu J, Hellmann MD, et al. Treatment outcomes of immune-related cutaneous adverse events. J Clin Oncol 2019;37(30):2746-2758. doi:10.1200/ JCO.18.02141.

4. Petrelli F, Grizzi G, Ghidini M, et al. Immune-related adverse events and survival in solid tumors treated with immune checkpoint inhibitors: a systematic review and meta-analysis. J Immunother. 2020;43(1):1-7. doi:10.1097/ CJ.0000000000000300.

5. Wongvibulsin S, Pahalyants $V$, Kalinich $M$, et al. Epidemiology and risk factors for the development of cutaneous toxicities in patients treated with immune checkpoint inhibitors: A United States population-level analysis. J Am Acad Dermatol. Published online 2021:1-10. doi:10.1016/j.jaad.2021.03.094.

6. Kalinich M, Murphy W, Wongvibulsin S, Pahalyants V, et al. Prediction of severe immune-related adverse events requiring hospital admission in patients on immune checkpoint inhibitors: study of a population level insurance claims database from the USA. J Immunother Cancer. 2021;9(3):e001935. doi: 10.1136/jitc2020-001935. PMID: 33789879; PMCID: PMC8016099.

Ethics Approval Study utilized de-identified data from a multiinstitutional registry and is exempt from IRB approval.

http://dx.doi.org/10.1136/jitc-2021-SITC2021.814 